\title{
Correction to: Improvement in Cognitive Function as Measured by NeuroTrax in Patients with Relapsing Multiple Sclerosis Treated with Natalizumab: A 2-Year Retrospective Analysis
}

Mark Gudesblatt ${ }^{1} \cdot$ Karl Wissemann $^{1} \cdot$ Myassar Zarif $^{1} \cdot$ Barbara Bumstead $^{1} \cdot$ Lori Fafard $^{1} \cdot$ Jeffrey Wilken $^{2}$. Karen Blitz ${ }^{1}$ - Marijean Buhse ${ }^{1}$. Sourav Santra ${ }^{3}$. Christophe Hotermans ${ }^{3} \cdot$ Lily Lee $^{3}$ (1)

Published online: 22 September 2018

(c) Springer Nature Switzerland AG 2018

\section{Correction to: CNS Drugs}

https://doi.org/10.1007/s40263-018-0553-1

An Online First version of this article was made available online at http://link.springer.com/journal/40263/onlineFirst/ page/1 on 24 August 2018. An error was subsequently identified in the article, and the following correction should be noted:

Author listing: The name of the second author, which previously read:

'Karl Wisseman'

Should read:

'Karl Wissemann'.

The original article has been corrected.

The original article can be found online at https://doi.org/10.1007/ s40263-018-0553-1.

Lily Lee

lily.lee@biogen.com

South Shore Neurologic Associates, Patchogue, NY, USA

2 Washington Neuropsychology Research Group LLC, Fairfax, VA, USA

3 Biogen, 225 Binney Street, Cambridge, MA 02142, USA 